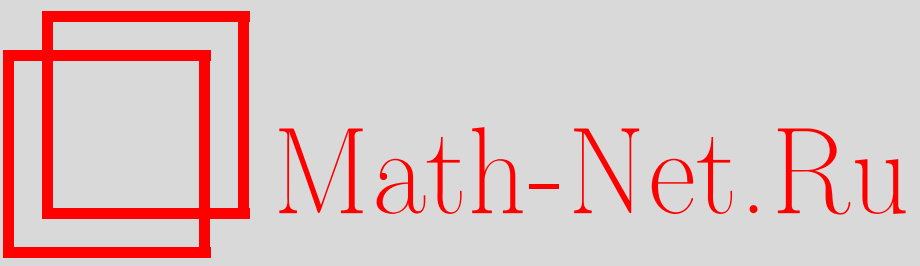

И. В. Баскаков, В. М. Бухштабер, Т. Е. Панов, Алгебры клеточных коцепей и действия торов, УМН, 2004, том 59, выпуск 3, 159-160

DOI: https://doi.org/10.4213/rm743

Использование Общероссийского математического портала Math-Net.Ru подразумевает, что вы прочитали и согласны с пользовательским соглашением

http://www.mathnet.ru/rus/agreement

Параметры загрузки:

IP: 54.209.52.79

26 апреля 2023 г., 05:24:00 


\title{
АЛГЕБРЫ КЛЕТОЧНЫХ КОЦЕПЕЙ И ДЕЙСТВИЯ ТОРОВ
}

\author{
И. В. БАСКАКОВ, В. М. БУХШТАБЕР, Т. Е. ПАНОВ
}

Дано доказательство изоморфизма алгебры целочисленных когомологий момент-угол комплекса $\mathscr{Z}_{K}[1]$ и Tor-алгебры кольца граней симплициального комплекса $K$. В основе его лежит построение клеточной аппроксимации диагонального отображения $\Delta: \mathscr{Z}_{K} \rightarrow \mathscr{Z}_{K} \times \mathscr{Z}_{K}$. В клеточных коцепях отсутствует функториальное ассоциативное умножение, так как в общем случае нельзя выбрать соответствующую клеточную аппроксимацию диагонали. Конструкция момент-угол комплекса является функтором из категории симплициальных комплексов в категорию пространств с действием тора. Мы показьваем, что в данном специальном случае предлагаемая клеточная аппроксимация диагонали ассоциативна и функториальна относительно отображений момент-угол комплексов, индуцированных симплициальньми отображениями. Кольцом граней комплекса $K$ на множестве вершин $[m]=\{1, \ldots, m\}$ назьвается градуированное факторкольцо $\mathbb{Z}[K]=\mathbb{Z}\left[v_{1}, \ldots, v_{m}\right] /\left(v_{\omega}: \omega \notin K\right)$ с $\operatorname{deg} v_{i}=2$ и $v_{\omega}=v_{i_{1}} \cdots v_{i_{k}}$, где $\omega=\left\{i_{1}, \ldots, i_{k}\right\} \subseteq[m]$. Пусть $B T^{m}$ - классифицирующее пространство для $m$-мерного тора со стандартным клеточным разбиением. Вводится клеточньй подкомплекс $D J(K):=\bigcup_{\sigma \in K} B T^{\sigma} \subseteq B T^{m}$, где $B T^{\sigma}=$ $\left\{x=\left(x_{1}, \ldots, x_{m}\right) \in B T^{m}: x_{i}=p t\right.$ при $\left.i \notin \sigma\right\}$. Используя данную клеточную структуру доказывается изоморфизм колец $H^{*}(D J(K)) \cong \mathbb{Z}[K]$ (см. [2; лемма 2.8]). Пусть $D^{2} \subset \mathbb{C}$ - единичньй диск; положим $B_{\omega}:=\left\{\left(z_{1}, \ldots, z_{m}\right) \in\left(D^{2}\right)^{m}:\left|z_{j}\right|=1\right.$ при $\left.j \notin \omega\right\}$. Момент-угол комплекс - это $T^{m}$-инвариантное подпространство $\mathscr{Z}_{K}:=\bigcup_{\sigma \in K} B_{\sigma} \subseteq\left(D^{2}\right)^{m}$. Как показано в [1; гл. 6], пространства $D J(K)$ и $\mathscr{Z}_{K}$ гомотопически эквивалентны пространствам, введенным в [3], что объясняет наши обозначения. Комплексы $\mathscr{Z}_{K}$ дают важньй класс действий тора. Пространство $\mathscr{Z}_{K}$ является гомотопическим слоем вложения $D J(K) \hookrightarrow B T^{m}$; оно возникает также как поверхность уровня отображения моментов при построении торических многообразий на основе симплектической редукции и как дополнение конфигурации координатных подпространств, определяемой комплексом $K$, см. [1; $\S 8.2]$.

Теорема 1. Имеет место функториальный по $K$ изоморфизм алгебр

$$
H^{*}\left(\mathscr{Z}_{K} ; \mathbb{Z}\right) \cong H\left[\Lambda\left[u_{1}, \ldots, u_{m}\right] \otimes \mathbb{Z}[K], d\right] \cong \operatorname{Tor}_{\mathbb{Z}\left[v_{1}, \ldots, v_{m}\right]}(\mathbb{Z}[K], \mathbb{Z})
$$

где в чентре стоит алгебра когомологий дифференциальной градуированной алгебры $c$ $\operatorname{deg} u_{i}=1, \operatorname{deg} v_{i}=2, d u_{i}=v_{i}, d v_{i}=0$.

В случае рациональных коэффициентов эта теорема была доказана в [4] применением техники спектральных последовательностей (см. также [1; теорема 7.6, проблема 8.14]). Наш новый метод доказательства использует построение алгебры клеточных коцепей. Недавно мы узнали, что другое доказательство теоремы 1 было независимо получено М. Францем [5; теорема 1.2].

ДоКАЗАТЕЛЬСТво теОРемы 1. Мы докажем лишь первый изоморфизм, так как второй вытекает стандартным образом из рассмотрения резольвенты Кошуля (детали см. в [1]). Введем дополнительную градуировку, положив bideg $u_{i}=(-1,2), \operatorname{deg} v_{i}=(0,2)$, и рассмотрим факторалгебру $R^{*}(K):=\Lambda\left[u_{1}, \ldots, u_{m}\right] \otimes \mathbb{Z}[K] /\left(v_{i}^{2}=u_{i} v_{i}=0, i=1, \ldots, m\right)$. Пусть $\varrho: \Lambda\left[u_{1}, \ldots, u_{m}\right] \otimes \mathbb{Z}[K] \rightarrow R^{*}(K)$ - каноническая проекция. Используя конечный аддитивный базис из мономов вида $u_{\omega} v_{\sigma}$, где $\omega \subseteq[m], \sigma \in K$ и $\omega \cap \sigma=\varnothing$ в алгебре $R^{*}(K)$ зададим аддитивное вложение $\iota: R^{*}(K) \rightarrow \Lambda\left[u_{1}, \ldots, u_{m}\right] \otimes \mathbb{Z}[K]$, удовлетворяющее соотношению $\varrho \cdot \iota=\mathrm{id}$. Покажем, что $\varrho$ индуцирует изоморфизм когомологий. Для этого построим оператор коцепной гомотопии $s$ такой, что $d s+s d=\mathrm{id}-\iota \cdot \varrho$. В случае $K=\Delta^{m-1}$ (полный симплекс) алгебра $\Lambda\left[u_{1}, \ldots, u_{m}\right] \otimes \mathbb{Z}[K]$ принимает вид $E=E_{m}=\Lambda\left[u_{1}, \ldots, u_{m}\right] \otimes \mathbb{Z}\left[v_{1}, \ldots, v_{m}\right]$, а алгебра $R^{*}\left(\Delta^{m-1}\right)$ изоморфна алгебре $R^{*}\left(\Delta^{0}\right)^{\otimes m}$, где $R^{*}\left(\Delta^{0}\right)=\Lambda[u] \otimes \mathbb{Z}[v] /\left(v^{2}=u v=0\right)$. Непосредственно проверяется, что для $m=1$ отображение $s_{1}: E^{0, *}=\mathbf{k}[v] \rightarrow E^{-1, *}$, определенное по формуле $s_{1}\left(a_{0}+a_{1} v+\cdots+a_{j} v^{j}\right)=\left(a_{2} v+a_{3} v^{2}+\cdots+a_{j} v^{j-1}\right) u$, является необходимой коцепной

Работа выполнена при поддержке грантов РФФИ 02-01-00659 и НШ-2185.2003.1. 
гомотопией. Далее по индукции мы можем предположить, что для $m=k-1$ оператор коцепной гомотопии $s_{k-1}: E_{k-1} \rightarrow E_{k-1}$ уже построен. Так как $E_{k}=E_{k-1} \otimes E_{1}, \varrho_{k}=\varrho_{k-1} \otimes \varrho_{1}$ и $\iota_{k}=\iota_{k-1} \otimes \iota_{1}$, отображение $s_{k}=s_{k-1} \otimes \mathrm{id}+\iota_{k-1} \varrho_{k-1} \otimes s_{1}$ является коцепной гомотопией между id и $\iota_{k} \varrho_{k}$. В случае произвольного комплекса $K$ алгебры $\Lambda\left[u_{1}, \ldots, u_{m}\right] \otimes \mathbb{Z}[K]$ и $R^{*}(K)$ получаются дополнительной факторизацией алгебр $E_{m}$ и $R^{*}\left(\Delta^{m-1}\right)$ по мономиальному идеалу. Оператор $s$ выдерживает эту факторизацию и определяет искомую коцепную гомотопию.

Рассмотрим клеточное разбиение комплекса $\left(D^{2}\right)^{m}$, при котором каждый диск $D^{2}$ разбивается на клетки $1, T$ и $D$ размерностей 0,1 и 2 . Таким образом, клетки из $\left(D^{2}\right)^{m}$ параметризуются словами $\mathscr{T} \in\{D, T, 1\}^{m}$. Сопоставим каждой паре $\sigma, \omega \subseteq[m], \sigma \cap \omega=\varnothing$ слово $\mathscr{T}(\sigma, \omega)$, имеющее букву $D$ на позициях с номерами из $\sigma$ и букву $T$ на позициях с номерами из $\omega$. По построению, $\mathscr{T}(\sigma, \omega)$ задает клетку из $\mathscr{Z}_{K} \subset\left(D^{2}\right)^{m}$ тогда и только тогда, когда $\sigma \in K$. Комплекс клеточных коцепей $C^{*}\left(\mathscr{Z}_{K}\right)$ имеет аддитивный базис из коцепей вида $\mathscr{T}(\sigma, \omega)^{*}$. Отсюда видно, что размерности градуированных компонент в $C^{*}\left(\mathscr{Z}_{K}\right)$ и $R^{*}(K)$ совпадают. Более того, отображение $g: R^{*}(K) \rightarrow C^{*}\left(\mathscr{Z}_{K}\right), u_{\omega} v_{\sigma} \mapsto \mathscr{T}(\sigma, \omega)^{*}$, является изоморфизмом дифференциальных градуированных модулей и индуцирует аддитивный изоморфизм $H\left[R^{*}(K)\right] \cong H^{*}\left(\mathscr{Z}_{K}\right)$. Для завершения доказательства построим клеточную аппроксимацию $\widetilde{\Delta}_{K}$ диагонального отображения $\Delta: \mathscr{Z}_{K} \rightarrow \mathscr{Z}_{K} \times \mathscr{Z}_{K}$ такую, что индуцированное умножение на клеточных коцепях совпадает с умножением в алгебре $R^{*}(K)$ в силу изоморфизма $g$. В случае $K=\Delta^{0}$ имеем $\mathscr{Z}_{K}=D^{2}$. Положим $z=\rho e^{i \varphi} \in D^{2}$ и определим $\widetilde{\Delta}: D^{2} \rightarrow D^{2} \times D^{2}$ по формуле

$$
\widetilde{\Delta}(z)= \begin{cases}\left(1+\rho\left(e^{2 i \varphi}-1\right), 1\right) & \text { при } \varphi \in[0, \pi], \\ \left(1,1+\rho\left(e^{2 i \varphi}-1\right)\right) & \text { при } \varphi \in[\pi, 2 \pi) .\end{cases}
$$

Легко видеть, что $\widetilde{\Delta}$ есть клеточное отображение, переводящее $\partial D^{2}$ в $\partial D^{2} \times \partial D^{2}$ и гомотопное $\Delta$ в классе таких отображений. Для соответствующего умножения в клеточных коцепях имеет место мультипликативньй изоморфизм $R^{*}\left(\Delta^{0}\right) \rightarrow C^{*}\left(D^{2}\right)$. Следовательно, и для $K=\Delta^{m-1}$ наше отображение $\widetilde{\Delta}:\left(D^{2}\right)^{m} \rightarrow\left(D^{2}\right)^{m} \times\left(D^{2}\right)^{m}$ задает мултипликативньй изоморфизм:

$$
f: R^{*}\left(\Delta^{m-1}\right)=\Lambda\left[u_{1}, \ldots, u_{m}\right] \otimes \mathbb{Z}\left[v_{1}, \ldots, v_{m}\right] /\left(v_{i}^{2}=u_{i} v_{i}=0\right) \longrightarrow C^{*}\left(\left(D^{2}\right)^{m}\right) .
$$

Из конструкции комплекса $\mathscr{Z}_{K}$ непосредственно вытекает, что ограничение отображения $\widetilde{\Delta}$ на $\mathscr{Z}_{K}$ дает клеточное отображение $\widetilde{\Delta}_{K}: \mathscr{Z}_{K} \rightarrow \mathscr{Z}_{K} \times \mathscr{Z}_{K}$. Следовательно, имеет место мултипликативное отображение $q: C^{*}\left(\left(D^{2}\right)^{m}\right) \rightarrow C^{*}\left(\mathscr{Z}_{K}\right)$. Рассмотрим коммутативную диаграмму

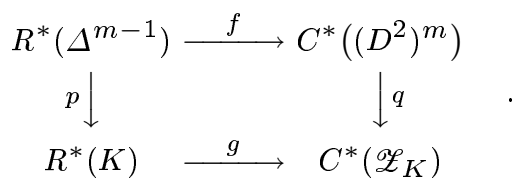

Используя, что $p, f$ и $q$ - кольцевые гомоморфизмы, а $p$ - эпиморфизм, мы получаем, что $g$ является кольцевым гомоморфизмом. Следовательно, $g$ - мультипликативньй изоморфизм.

\section{СПИСОК ЛИТЕРАТУРЫ}

[1] V.M. Buchstaber, T.E. Panov. Torus Actions and Their Applications in Topology and Combinatorics. Providence, RI: Amer. Math. Soc., 2002. (Univ. Lecture Ser. V. 24.) [2] В.М. Бухштабер, Т.Е. Панов // Зап. научн. семинаров ПОМИ. 2000. Т. 266. C. 29-50. [3] M. Davis, T. Januszkiewicz // Duke Math. J. 1991. V. 62. № 2. P. 417-451. [4] B. М. Бухштабер, Т.Е. Панов // Труды МИРАН. 1999. Т. 225. С. 96-131. [5] M. Franz. On the integral cohomology of smooth toric varieties // arXiv:math.AT/0308253. 